In der Rubrik „Literatur kompakt" werden die wichtigsten Originalarbeiten aus der internationalen Fachliteratur referiert.

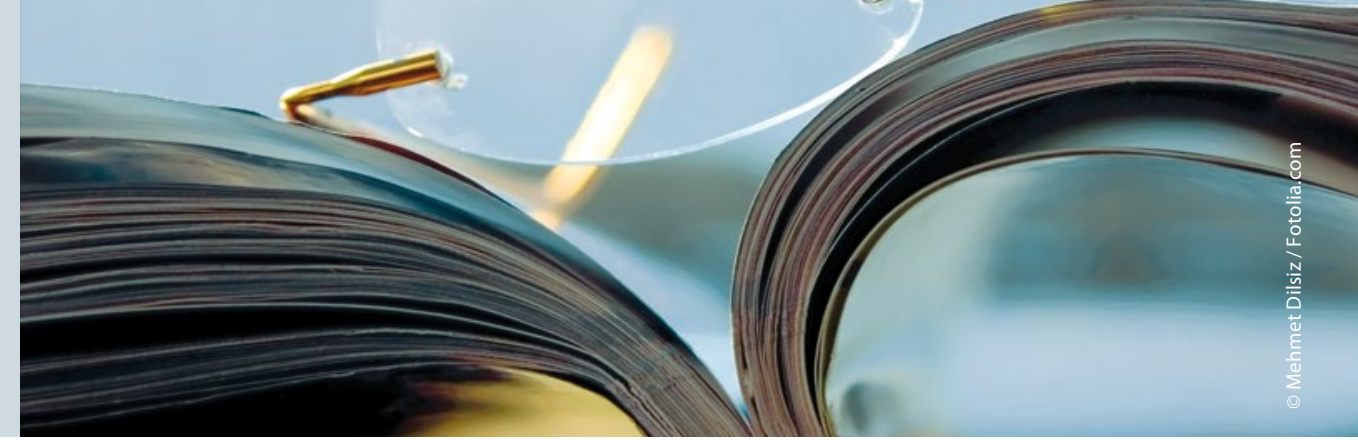

\section{Urogenitalbilharziose auch auf Korsika}

Die Urogenitalbilharziose galt bislang als eine auf tropische und subtropische Länder mit schlechter Hygiene beschränkte Infektionskrankheit. Durch den seit 2013 anhaltenden Ausbruch von Bilharziose auf der beliebten Ferieninsel Korsika muss auch in Deutschland mit importierten Erkrankungen von Urogenitalbilharziose gerechnet weden.

$\mathrm{D}$ ie Bilharziose ist eine durch Trematoden der Gattung Schistosoma hervorgerufene parasitäre Erkrankung. Obgleich nach Schätzungen weltweit 230 Millionen Menschen in tropischen und subtropischen Ländern von dieser Infektion betroffen sind, wird sie zu den vernachlässigten Erkrankungen gerechnet. Der Erregerzyklus ist komplex und benötigt Süßwasserschnecken als Zwischenwirt. Bei Mädchen und Frauen führt die Bilharziose zu ulzerierenden und papillomatösen Veränderungen der Vulva, zu fibrösen Massen des Uterus sowie der Adnexe, zu Salpingitis und als Folge des Tubenverschlusses zur Infertilität. In der Schwangerschaft kommt es zum Befall der Plazenta, wodurch das intrauterine Wachstum des Fötus beeinträchtigt werden kann. Die Blasenbilharziose ist durch Hämaturie, Dysurie, Pollakisurie und perineale Schmerzen charakterisiert.

Im Sommer 2013 brach die Urogenitalbilharziose mit mindestens 120 bestätigten Fällen auf Korsika aus. Betroffen waren neben Einheimischen auch Touristen, darunter deutsche Urlauber. Als Ansteckungsquelle wurde der bekannte Fluss Cavu identifiziert, der wegen seiner vielen Lagunen ein beliebtes Badeziel ist. Die Behörden reagierten mit einem Badeverbot, das im April 2015 aufgehoben wurde. Im Sommer $2015 \mathrm{kam}$ es erneut zu Infektionen, was auf eine

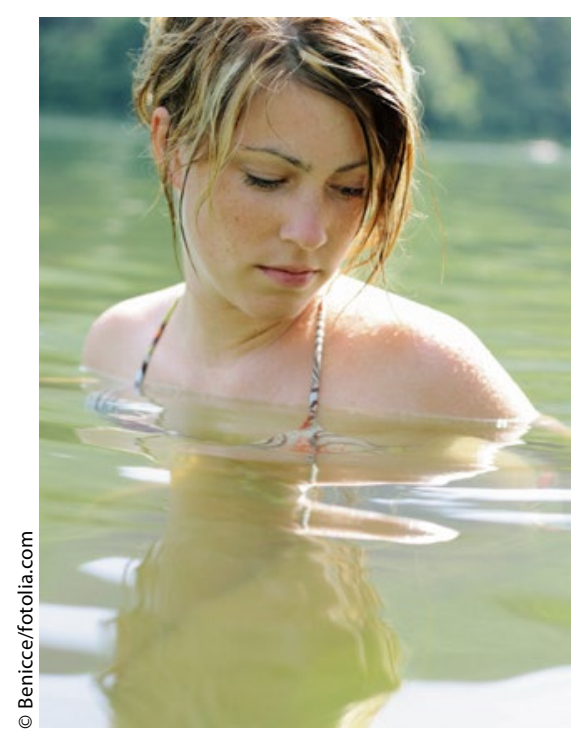

Die Tropen müssen es gar nicht sein: Schistosomen fühlen sich inzwischen auch im europäischen Süßwasser wohl. Das kann zur Gefahr beim Baden werden.

Persistenz der Schistosomen in Wasser-

In einer jetzt vorgelegten Untersuchung wurde gezeigt, dass Wasserschnecken (Bulinus truncatus) aus dem Fluss mit Patientenisolaten von Schistosoma haematobium infizierbar waren. Zudem waren diese Schnecken für Hybridformen zwischen menschlichen und Rinder-Schistosomen empfindlich, was auf schnecken hindeutete. eine Bedeutung von Rindern in dem neu entstandenen Kreislauf hinweist. Die molekulargenetische Analyse dieser Isolate konnte belegen, dass die Schistosoma-haematobium-Stämme aus dem Senegal in Westafrika stammen. Die Autoren folgern, dass die Parasiten durch in Westafrika Infizierte nach Korsika kamen und Urinieren in den Fluss zur Infektion der Wasserschnecken führte.

Boissier J et al. Outbreak of urogenital schistosomiasis in Corsica (France): an epidemiological case study. Lancet Infect Dis. online May 16 2016, doi: http://dx.doi.org/10.1016/S14733099(16)00175-4

\section{Kommentar}

Die Untersuchungen zeigen, wie leicht und schnell sich die urogenitale Bilharziose auch in neuen Arealen ausbreiten kann. Da die ökologischen Gegebenheiten wie auch das Verbreitungsgebiet der Vektorwasserschnecken in allen Mittelmeerländern ähnlich ist, besteht somit potenziell die Gefahr weiterer Bilharziose-Ausbrüche in anderen europäischen Regionen sowie das Risiko einer stabilen Etablierung des Erregerzyklusses. Bei unklaren Fällen von Hämaturie sowie ulzerösen und papillomatösen Veränderungen der Vulva sollte daher bei einer entsprechenden Reiseanamnese auch an eine Bilharziose gedacht werden. Diese Möglichkeit sollte bei passender Symptomatik auch bei der Untersuchung von Migrantinnen aus endemischen Ländern erwogen werden.

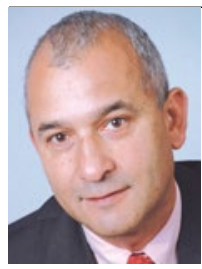

Prof. Tino F. Schwarz Zentrallabor, Stiftung Juliusspital Würzburg 